\title{
Discussion on the Construction and Sustainable Development of Urban Ecological Gardens
}

\author{
Haiyang Sun \\ Guangdong Academy of Agricultural Sciences, Guangzhou, China.
}

How to cite this paper: Haiyang Sun. (2021) Discussion on the Construction and Sustainable Development of Urban Ecological Gardens. Advance in Sustainability, 1(1), 1-6. DOI: 10.26855/as.2021.07.001

Received: June 2, 2021

Accepted: June 27, 2021

Published: July 14, 2021

*Corresponding author: Haiyang Sun, Guangdong Academy of Agricultural Sciences, Guangzhou, China.

\begin{abstract}
The Chinese culture is extensive and profound, which has been enduring for thousands of years, and contains rich and diverse art and culture, garden art is one of them. In terms of the development history of garden art, it has a long history. In the contemporary landscape architecture construction, ecological landscape architecture has attracted great attention from all walks of life, and it is also the trend of landscape architecture construction. In the ecological park forest, more attention should be paid to ecological protection and ecological balance, taking into account the beautification of the environment and the reduction of ecological environmental disasters, and vigorously advocating appropriate measures according to local conditions, suitable planting, ecological protection, and the benign circular development of biological chain, so as to achieve the goal of sustainable development. In recent years, as the change of age society, natural environment worsened than before, for now, these problems have been on the reflection, actively explore in the reflection, with the reality of our country in the exploration analysis of problems existing in the urban landscape construction, based on this, puts forward the corresponding solutions, promotes the good construction and sustainable development of ecological gardens.
\end{abstract}

\section{Keywords}

Ecological Garden, Construction, Sustainable Development, Garden Art, The Ecological Environment

\section{Introduction}

In today's era, in order to promote the sustainable development of the city, it is necessary to pay attention to the ecological balance. Therefore, in the urban garden, the exploration of sustainable development of ecological green road is particularly critical, which has attracted the attention of all walks of life. Garden is very important in urban ecology, and occupies an important position in urban ecology. In order to take urban ecology as the first priority, it is necessary to abandon the concept of beauty and enter a new era of ecological garden. This paper will discuss the ecological garden, discuss its current status and existing problems, and put forward targeted solutions, so as to realize the construction and sustainable development of ecological garden.

\section{Scientific connotation of ecological gardens}

First, refers to on the basis of landscape ecology landscape, through scientific ways and means, to establish a certain amount of artificial plant community, and on the artificial plant community in the process of design, both need to consider the spatial structure factor, also need to consider the time factor, etc., thus to construct a good ecological environment, realize the sustainable development of ecological landscape. 
Second, ecological garden is based on the principle of ecology, to make full use of all kinds of green plants, make full use of all kinds of energy, such as solar energy, make full use of solar energy, convert it into chemical energy, maximize the use of solar energy, and then to local climate regulation. At the same time, through a variety of green plants, it can also purify toxic gases in the environment, reduce noise, and achieve ecological balanced development.

Third, beautify the landscape. Pay attention to the artistic level design in the green environment, improve the aesthetic degree of the ecological garden, make the ecological garden has the value of viewing and visiting, increase its social benefits, meet people's cultural needs, and provide people with entertainment, leisure and elegant green environment. In ecological forest ecology is one of the most important, it is the basis for the ecological garden, on this basis, also need to be integrated into the landscape ecology, landscape, urban ecological theory, etc., for the influence of the landscape architecture and urban green space ecology system are studied, on the basis of ecology, pay attention to the diversity of creatures, on the basis of constructing urban communities, then make the city has a stable ecological garden, promote the coordinated development of urban ecological garden, to ensure that the city ecology is in a state of balance. In the ecological park forest, more attention is paid to the relationship between human and nature and the harmonious development between them, in order to achieve sustainable development. In today's era, with the improvement of people's living standards, people have greater desires and demands in addition to the basic needs of life. However, the natural supply is limited, and the contradiction between the two is becoming more and more intense. But through the way of ecological garden, is conducive to alleviate this contradiction, promote the virtuous cycle of the ecosystem, which is conducive to promoting economic and social development, but also conducive to promoting the balanced development of the ecology.

\section{Prospects of ecological gardens}

\subsection{The demand for ecological gardens grows day by day}

With the continuous development of economy and society, the improvement of people's living standards, food and clothing is not the only pursuit of people, people have higher expectations of the society, pay more attention to the ecological level of the environment, pay more attention to their own living environment. In order to transform people's living environment and provide people with an ecological living environment, it is necessary to strengthen the development of ecological gardens. In terms of the development of ecological gardens, many cities attach great importance to the construction of ecological gardens. The city of Warsaw in Poland is known as the "Green Capital of the World". There are 65 parks in the city. The forest in the suburbs is as high as one million mu, and the green land is 90 per capita. Moscow City also attaches great importance to the construction of ecological gardens. There are 11 forest areas, 84 parks, 100 street parks and 720 street parks. In the urban area, green land accounts for a large proportion, up to $40 \%$ of the total area. Australia's capital, Canberra, has even more green space, about 60 percent of the city's total area. In the campaign of "Garden City" in Singapore, there are strict requirements for urban greening. The green belts between roads and buildings have made specific requirements. The whole city has good air and is full of greenery. Compared with the above countries, China's urban green space is very little, still at a low level, the per capita green space is low.

In addition, due to the environmental awareness of Chinese residents, even though there are few green areas in China, these green areas are still covered by urban construction, making the deterioration of the ecological environment more and more serious. In this case, it is necessary to strengthen the improvement of ecological environment and popularize ecological gardens. Nowadays, all countries are paying attention to the sustainable development of the ecological environment. In this case, it is necessary to pay attention to the sustainable use of plants, so as to enable the better development of all species and provide them with a good and sustainable development of the ecological environment. In this process, ecological garden plays an important role. Taking the environment as the starting point, the ecological park pays attention to the coordinated development of natural environment and social environment, so as to promote the benign and balanced development of ecology.

\subsection{Maturity of related disciplines}

In the pace of the development of The Times, people have a deeper understanding of ecological gardens, and at the same time, all aspects of them have been fully studied. In this process, the research direction has been expanded, mainly including the following two research directions: First, small-scale research. In this research direction, it mainly focuses on small-scale studies, including the study of ecological gardens in urban construction and the influence of ecological gardens in a certain area of the city. In the study, mainly study the ecological garden in a small scale, can the specific study the influence of the ecological landscape of city, to strengthen the awareness of ecological garden, realize the importance of ecological garden, understanding the ecological landscape to the positive role of the city, to guarantee the sustainable development of ecological garden of benign, promote the development of the ecological balance. Second, the superscale of research. In this research direction, ecological gardens are mainly studied from a global perspective. To study the significance of ecological gardens to 
global warming, and to realize the significance of sustainable development of economy, society and environment. Study on the importance of ecological garden construction in the environment, on this basis, promote the further development of ecological garden construction.

\section{The concept of sustainable development}

Opportunities and crises coexist in the development of the 21st century. People's living standards have been qualitatively improved, but at the same time, the concept of sustainable development has been frequently mentioned in various fields. This means that with the rapid economic development, there is also a certain cost, and we need to ensure the economic development at the same time, as far as possible to reduce the cost, to achieve sustainable economic and social development. As far as sustainable development is concerned, it means that in the process of economic and social development, we should not only meet the needs of the present generation, but also avoid harming the rights and interests of future generations and threatening the needs of future generations. This concept includes not only sustainability, but also fairness and systematic Ness. This concept is scientific in nature. For a city, sustainable development is very important, and even plays a decisive role in the survival of a city. However, its factors are complicated, including historical factors, economic and social factors, etc. In the process of construction of ecological garden, it is necessary to adhere to the scientific and reasonable ecological planning, respecting nature, protecting biodiversity, to ensure that the urban ecological resources, guarantee the city has a good water circulation, not destruction, does not affect the survival of the environment and habitat, the minimum impact on the nature of all kinds of animals and plants under the situation of ecological landscape construction, protecting people's ecological environment.

\section{Problems in the construction of ecological gardens}

\subsection{Problems of planting soil}

With the further development of urbanization, engineering construction can be seen everywhere. In engineering construction, domestic garbage or some waste materials are usually used as foundation filling materials. In some areas, the terrain is quite different, which means that in order to fill the foundation, more waste and garbage are needed. But in the process, there is no soil at all underneath the foundation, making it difficult for plants to live and grow. Although some cities in the face of this situation, will cover the soil on the waste or filling garbage, but this method has little effect, neither can cure the root, let alone cure the symptoms, cannot make the problem of plant growth can be effectively solved. In addition, there are some other factors, some greening workers in the process of planting plants, in order to reduce the workload, as soon as possible to complete the task, the pit is shallow or small, resulting in the root of the plant in the process of growth cannot be completely stretched, there is a slowdown phenomenon, is not conducive to the healthy growth of plants. And after planting, the plants are left to themselves without fertilization or watering, which makes the survival rate of the plants low. In the harsh conditions, although some young plant to survive, but because of the growth in the process of the lack of adequate fertilizer and water, its shape is very thin, and resistance is poor, not frequent diseases and insect pests, which not only affect individual plants, but also can make the cross infection occurs between plants, thus affect the entire ecological garden of vegetation. In a word, ecological garden is the construction of artificial creation and planning of plant groups. In the process of building ecological garden, in order to ensure the survival rate of plants and improve the quality of soil, it is necessary to do a good job in the maintenance of trees after planting, so as to achieve the sustainable development of ecological garden.

\subsection{The quality of plant seedling cultivation is uneven}

In the construction of ecological gardens, there are also problems in purchasing Miao source. Because most of the relevant departments select seedlings from multiple seedling garden bases rather than from one seedling garden base in the process of purchasing seedling sources, the quality of seedling culture of plants is different in this case. As far as the seedling source itself is concerned, there are differences in quality and specification, so the greening results after planting them also have certain differences. And the growth of plants is a relatively long process, cannot be seen immediately after cultivation results. But as the plants grow and flourish, the gap between the plants will become increasingly significant, which will affect the beauty of the garden. As a practical matter, whenever the golden age of plant cultivation, the relevant personnel will buy the seedlings through various channels, which leads to the lack of consistency of the sources of seedlings, but rather complex. As far as the source of seedlings is concerned, it is not always abundant, but sometimes absent. In the lack of seedlings resources period, the relevant personnel only pay attention to the number of seedlings, ignoring the quality of seedlings, focusing on buying seedlings, regardless of their quality. In this case, only the number of seedlings can be guaranteed, it is difficult to ensure the quality of seedlings. And sometimes in the process of purchasing seedlings, there is no consideration of all aspects of the factors, neither consider the adaptability of seedlings to the environment, nor pay attention to the reasonable placement of seedlings, at the same time, there is no attention to the situation of infestation of seedlings, leading to cross-infection of seedlings. In order to 
better build ecological gardens, it is necessary to avoid the above situation as far as possible, so we can consider combining all the forces to establish a local nursery stock supply base.

\subsection{Problems exist in external factors of plant growth}

The ubiquitous presence of plants in our daily lives has led us to believe that plants are hardy and can stand up even when exposed to wind and rain. But the fact is not so, a variety of external factors, will also have an impact on plant growth. Hot weather can make the plant cannot get enough water, the water consumption of the plant, and then make the plant die of drought. Rainy weather may make the plants get too much water, long-term immersion in the rain, plants cannot absorb in time, resulting in waterlogging death of plants. In addition, different plants differ in their ability to adapt to climate. Some plants need to be grown in subtropical areas; some plants need to be grown in the tropics; some plants need to be grown in temperate regions. Only when plants are planted in an area suitable for their growth can they grow healthily and flourish. And so on, many external factors can affect plant growth. China is divided into the south and the north, and there are great differences in precipitation and climate between the north and the south. In terms of the specific weather conditions in the north, there is less precipitation and the climate is relatively dry. This kind of natural condition is relatively harsh, is not favored by the majority of plants, at the same time suitable for the northern ecological garden construction of plant species are less. Ecological garden is not only conducive to people's leisure and enjoy the natural and green environment, but also has the overall performance effect. Compared with the southern region, the conditions in the northern region are more severe for the growth of plants. The higher the latitude in the northern region, the less precipitation, so there are fewer plant species that can survive in the local area, which makes the ecological garden tend to use several kinds of plants in the construction process, resulting in a single greening situation. Even if measures such as species introduction are taken, due to improper consideration, the introduced species repel the environment, the survival rate is not high, and it is easy to destroy the overall coordination of the original body, which is not conducive to improving the greening effect of ecological gardens. Therefore, in the construction of ecological gardens, it is necessary to pay attention to the integration of suitable multi-species plants, and at the same time to reflect the historical background of the city as much as possible.

\section{Measures taken in the construction of ecological gardens}

\subsection{Adhere to the ecology of ecological gardens}

In ecological garden forest, ecology is very important, running through the construction of ecological garden. In today's booming economic era, people's living standards are increasing day by day, so the public pays less and less attention to quantity and quality, but more attention to environmental protection. For this society, environmental protection is crucial. Environmental protection represents the sustainable development of the society and the life our children and grandchildren will lead. Therefore, environmental protection is of profound significance. And environmental protection and ecology are inseparable, in the construction of ecological gardens, we must pay attention to ecology.

In the construction of ecological garden, it is necessary to pay attention to ecology. The construction of ecological gardens is not only for people's leisure and beauty, but also for optimizing the environment and providing people with a better life. Therefore, in the construction of ecological gardens, we should increase the area of green land as much as possible, give full play to the role of green plants, purify the air through photosynthesis, so that people can breathe cleaner air; Reduce noise, so that people get a quieter living environment. So on, in the construction of ecological gardens, we should excavate the ecological role of ecological gardens from all aspects, so as to make them contribute to the green environment and realize the sustainable development of ecological gardens.

\subsection{Scientific planning of the structure of garden layout}

In terms of the concrete construction of ecological gardens, there are various problems. For example, in the construction of urban gardens, the planning is lack of order, and the layout is not scientific and reasonable, too scattered. In terms of landscape, too much artificial carved landscape, too little natural landscape, etc. In the process of the construction of ecological gardens, we should adhere to the principle of ecology first and plan the ecological gardens with an eye to the whole. At the same time, in this process, it is also necessary to take the ecological balance as the starting point, pay attention to the overall ecological environment of the garden, join the natural environment in the garden, adhere to the principle of garden combination, scientific and reasonable arrangement of the seedlings inside the garden, enhance its ornamental value and the role of green environment. In this way, the land utilization rate can be greatly improved, the limited land in the city can be used to the maximum extent, and the greening area of the garden can be expanded. In addition, in the process of landscape planning of city, need according to the conditions of the plant species, carry on the reasonable configuration, the analysis of features of various plant species, and then pointed to its counterpart in planting, form a benign ecological group, so that we can create good ecological landscape, 
avoid landscape of oneness, Enhance the appreciation of ecological gardens, so that ecological gardens with natural nature.

\subsection{Reasonable planting based on the characteristics of species}

In the construction of ecological gardens, shrubs, herbs, lianas and other plant groups are widely used. These plant groups have different characteristics in nature and have their own advantages, which cannot be replaced by each other. Therefore, in the ecological garden construction, the need to fully consider the location of the garden, but also need to consider the characteristics of cultivated plants, in the process of buying seedlings cannot have blindness, optional purchase, a large number of purchases. Instead, species similar to the local living environment should be cultivated according to the specific conditions of the city in which the garden is located, on this basis, scientific and reasonable collocation of trees, shrubs, herbs and so on. In short, in the construction of urban ecological gardens, it is necessary to give full consideration to the ecological environment of the place, identify the plants suitable for local growth, consider the actual situation of the local, whether it is possible to transplant exotic species, and then make a reasonable configuration of the garden. In this way, it is not only beneficial to improve the survival rate of plants, but also beneficial to reduce the maintenance cost after plant cultivation, reduce the cost of garden construction, and reflect the value of ecological garden more.

\subsection{Select characteristic plant communities based on urban characteristics}

Each city is different, and each city has its own characteristics. Therefore, in the process of ecological garden construction, in addition to ecological factors, local characteristics need to be fully considered, and urban characteristics should be integrated into plant cultivation. At the same time, in the process of selecting seedlings, we should also choose plants with unique local characteristics, because these seedlings are representative of the local area, and it is very easy to grow in the local climate, with a high survival rate, and also has an extremely fast growth rate. In addition, plants with local characteristics have a high adaptability in the local area, which is not only a manifestation of local characteristics, but also has a strong ability to resist pests and diseases, and is less disturbed by the environment. Choosing plants with local characteristics is more conducive to the construction of ecological gardens, creating a good ecological environment, giving full play to the role of ecological gardens, creating a better living environment for people, and realizing the sustainable development of ecological garden construction. In the construction of ecological gardens, although the need to pay attention to the characteristics of local native plants, but also need according to the actual situation, the appropriate introduction of foreign plant species, so that we can make the ecological landscape vegetation species richer, improve ecological gardens can view and admire a gender, make the ecological landscape more beautiful, to the local residents show except for the native plant communities.

\subsection{Maintain the diversity of ecological garden species}

In a city, residents are the main body and the master of the city. Therefore, in the process of ecological garden construction, we must fully consider the residents' psychological activities to meet the requirements of urban residents for ecological gardens. In the process of ecological garden construction, we need to adhere to the people-oriented, the needs of urban residents as the premise of garden construction. In the process of ecological landscape construction, need to be in all aspects to consider of the diverse needs of residents, not only to make the ecological garden is beneficial to people's physical and mental health development, but also need to consider whether ecological landscape makes people get a good psychology and visual perception, the construction of characteristic landscape, let people can gain happiness from landscape, it fully reflects the humanism of ecological garden construction. In the process of design of the ecological garden, no matter how strange idea, but is must to residents demand first, meet the convenience of residents in entertainment, leisure, increase the time duration of the residents in the landscape, let every resident can get in the garden of the soul are calm, make the ecological landscape in their heart of a Promised Land, let them willing to walk into the ecological garden. Only in this way can we say that the construction of ecological gardens is a successful construction and can really attract people to stay in the construction of ecological gardens. In short, in the process of ecological garden construction, we need to adhere to the people-oriented, based on people's needs for ecological garden construction, to meet people's various needs for garden construction.

\section{Conclusion}

In the development of The Times, the construction of ecological gardens is more and more important, which plays an important role in the rapid development of the city. Ecological garden is not only conducive to providing people with a beautiful environment, leisure and entertainment places, but also can green the environment, so that people have a better living and living environment. However, in terms of the actual situation of ecological garden construction, it is not optimistic, there are still a variety of problems, this paper discussed these problems, targeted put forward the solution measures, in order to promote the further development of ecological garden construction, promote the green development of China's cities, promote the sustain- 
able development of society.

\section{References}

[1] Guo Dongli, Li Mei. (2019). Discussion on the quality management of landscaping [J]. Sichuan Forestry Science and Technology, 2019, 20(1): 64-68.

[2] Wang Hao, Zhao Yongyan. (2020). Concepts and ideas of urban ecological garden planning [J]. Journal of Nanjing Forestry University, 2020, 24(5): 85-88.

[3] Deng Baozhong, et al. (2019). Landscape ecological construction and urban sustainable development [J]. Protection Forest Science and Technology, 2019, (5): 64-66. 